\title{
One-year outcomes following implantation of second-generation trabecular micro-bypass stents in conjunction with cataract surgery for various types of glaucoma or ocular hypertension: multicenter, multi-surgeon study
}

This article was published in the following Dove Medical Press journal: Clinical Ophthalmology

Colin I Clement ${ }^{1-4}$

Frank Howes ${ }^{5}$

Alexandros S loannidis ${ }^{6}$

Michael Shiu ${ }^{7}$

David Manning ${ }^{8}$

'Eye Associates, Sydney, NSW, Australia; ${ }^{2}$ Fairfield Eye Surgery, Fairfield, NSW, Australia; ${ }^{3} \mathrm{Glaucoma}$ Unit, Sydney Eye Hospital, Sydney, NSW, Australia; ${ }^{4}$ Discipline of Ophthalmology, The University of Sydney, Sydney, NSW, Australia;

${ }^{5}$ Eye \& Laser Centre Gold Coast, Southport, QLD, Australia; ${ }^{6}$ Vision Eye Institute, Melbourne, VIC, Australia; ${ }^{7}$ Laser Sight Laser Cataract \& Lens Specialists, Sydney, NSW, Australia; ${ }^{8}$ Hunter Cataract \& Eye Centre, Sydney, NSW, Australia
Correspondence: Colin Clement Eye Associates, Level 4, I87 Macquarie Street, Sydney 2000, NSW, Australia

Tel +6I 292479972

Email colinandkylie@me.com
Purpose: To assess the utility and safety of implanting two second-generation trabecular micro-bypass stents following cataract surgery in eyes with mild to advanced glaucoma or ocular hypertension (OHT).

Patients and methods: Retrospective study of iStent ${ }^{\mathbb{B}}$ inject trabecular micro-bypass implantation with cataract surgery by five surgeons at five sites in Australia. Eyes had mild to advanced glaucoma (predominantly primary open-angle, appositional angle-closure, or pseudoexfoliative glaucoma) or OHT and cataract requiring surgery. Effectiveness measures included intraocular pressure (IOP); medication burden; and proportions of eyes with no medications, $\geq 2$ medications, stable or decreased medications vs preoperative, and IOP $\leq 18 \mathrm{mmHg}$. Safety measures included visual acuity, cup-to-disc ratio (CDR), visual field (VF), complications, adverse events, and secondary surgical interventions. Patients have been followed for 12 months, and follow-up is ongoing.

Results: Of the 290 total eyes that underwent surgery, 165 eyes had 12-month outcomes at the time of data collection and are included in this report. In these eyes, mean Month 12 IOP reduced by $23.2 \%$ from $18.27 \pm 5.41 \mathrm{mmHg}$ preoperatively to $14.04 \pm 2.98 \mathrm{mmHg}(P<0.001)$, with $95.8 \%$ of eyes achieving Month 12 IOP of $\leq 18 \mathrm{mmHg}$ vs $60.6 \%$ preoperatively. Mean number of medications at 12 months decreased by $71.5 \%, 0.47 \pm 0.95$ vs $1.65 \pm 1.28$ preoperatively; $76.4 \%$ of eyes were on zero medications vs $17.6 \%$ preoperatively $(P<0.001) ; 14.5 \%$ of eyes were on $\geq 2$ medications vs $46.7 \%$ preoperatively $(P<0.001)$; and $98.2 \%$ of eyes maintained or reduced medications vs their preoperative regimen. Favorable safety included no stent-related intraoperative complications; limited and transient postoperative adverse events; and stable CDR, VF, and visual acuity. Three eyes with more advanced disease underwent additional glaucoma surgeries.

Conclusion: iStent inject implantation with cataract surgery significantly and safely reduced medications and IOP in eyes with various types and severities of glaucoma within a multicenter, multi-provider, real-life setting.

Keywords: microinvasive glaucoma surgery, MIGS, glaucoma, iStent inject, intraocular pressure, second-generation, medication, safety

\section{Introduction}

Microinvasive glaucoma surgery (MIGS) has become a viable option for the treatment of mild to moderate glaucoma, with increasing use particularly over the past decade. ${ }^{1}$ 
Trabecular bypass microstents can be inserted ab interno into the trabecular meshwork with minimal trauma through a small peripheral clear corneal incision, or through the phacoemulsification incision after cataract surgery. Randomized, controlled trials have shown significant reductions in both intraocular pressure (IOP) and medication use after implantation of one or more microstents in conjunction with cataract surgery, beyond the reductions achieved with cataract surgery alone. $^{2-4}$

Although trabeculectomy traditionally has been the gold standard incisional surgical treatment for elevated IOP, a recent survey by the American Glaucoma Society shows substantial use of MIGS in patients with glaucoma undergoing cataract surgery. In this survey, cataract surgery was combined with trabeculectomy by $24 \% \pm 23 \%$ of surgeons, while MIGS procedures were chosen by $22 \% \pm 27 \%$ of surgeons, in patients with primary openangle glaucoma (POAG) and visually significant cataract. ${ }^{11}$ Implantation of MIGS devices can decrease patient reliance on pharmacologic eye drops, whose utility often is limited by side effects, ${ }^{5,6}$ suboptimal adherence in the great majority of patients, and improper application..$^{7-9}$ MIGS surgery also spares the conjunctiva and avoids filtering blebs, improving patient safety, and quality of life. ${ }^{10}$

The iStent ${ }^{\circledR}$ trabecular micro-bypass stent (Glaukos Corporation, San Clemente, CA, USA) was the first MIGS device approved for use in the US, as well as in Australia (the site of the present cohort). The stent is implanted ab interno into Schlemm's canal, bypassing the trabecular meshwork and creating a path from the anterior chamber to the canal to improve aqueous outflow. The more recently developed second-generation iStent inject trabecular micro-bypass stent system allows surgeons to inject two stents without removing the inserter from the anterior chamber.

Several prior studies have documented the effectiveness and safety of iStent inject either with or without concomitant cataract surgery in eyes with open-angle glaucoma. These studies have documented sustained IOP reductions of $\sim 25 \%-40 \%$ versus medicated baseline, together with sizable decreases in medication burden. ${ }^{12-16}$ The use of iStent inject with concomitant cataract surgery was the subject of a recent multicenter prospective randomized clinical trial (Glaukos Corporation, NCT \#00323284), which led to the US Food and Drug Administration approval of the device in mid-2018.

As more surgeons adopt MIGS, and specifically incorporate iStent inject, it is important to understand the results they can expect in a routine clinical setting where the population is heterogeneous and rigid inclusion/exclusion criteria are not appropriate. These expectations are relevant not only for surgeons, but also for patients, as patients with realistic expectations for their surgical outcome are more likely to be happy with their surgical experience. ${ }^{17}$ In the current report, we present a large retrospective study of patients from five sites and five surgeons in Australia with outcomes through 12 months following iStent inject implantation in conjunction with cataract surgery.

\section{Patients and methods}

This retrospective outcomes assessment consists of data pooled from five surgeons at five different ophthalmology centers in Australia. The clinical records were reviewed to identify eyes that underwent iStent inject implantation together with cataract surgery between January 2016 and July 2017. The retrospective review was approved by the Royal Australian and New Zealand College of Ophthalmology Human Research Ethics Committee. Allowable diagnoses included POAG, appositional angle-closure glaucoma (ACG) or ACG suspects, pseudoexfoliative glaucoma (PXG), normal-tension glaucoma (NTG), pigmentary glaucoma, combined-mechanism glaucoma, and ocular hypertension (OHT). Eyes with ocular comorbidities other than glaucoma and cataract, eyes with active ocular inflammation, and eyes with synechial angle closure were not eligible for iStent inject surgery. Postoperative effectiveness measures included IOP and medication burden, and proportional analyses of eyes on no medications, eyes on $\geq 2$ medications, eyes with stable or decreased medication burden vs preoperative, and eyes with IOP $\leq 18 \mathrm{mmHg}$. Safety measures included corrected distance visual acuity (CDVA), cup-to-disc ratio (CDR), visual field (VF), surgical complications, postoperative adverse events, and secondary surgical interventions.

The iStent inject system contains two heparin-coated titanium stents that are fenestrated to provide a bypass for aqueous outflow through the trabecular meshwork (Figure 1). Following uncomplicated phacoemulsification cataract surgery, iStent inject implantation is completed as follows. Under gonioscopic visualization, the injector is inserted through the phacoemulsification incision and advanced to the trabecular meshwork, where the retraction button allows the insertion sleeve to pull back and expose the trocar tip. The microstents then are placed at least 2 clock hours apart through the trabecular meshwork and into Schlemm's canal. Once the positioning of the microstents is verified, the viscoelastic is removed, the eye is irrigated with balanced salt solution, and wound patency is assured. 

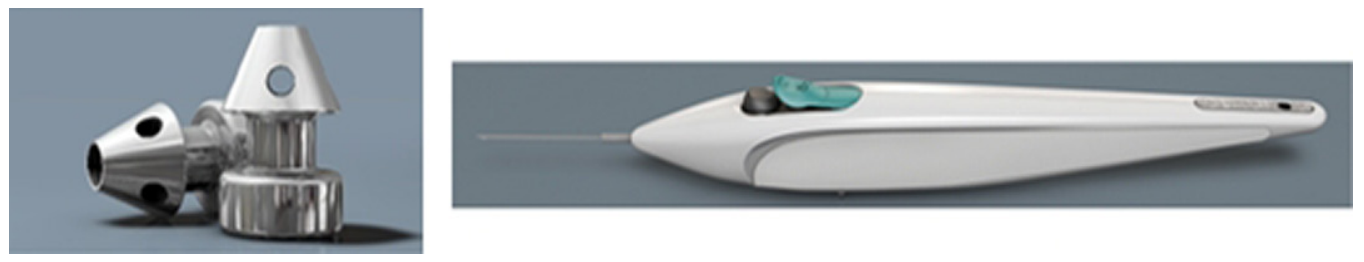

Figure I iStent ${ }^{\circledR}$ inject and iStent ${ }^{\circledR i n j e c t ~ t r a b e c u l a r ~ m i c r o-b y p a s s ~ s t e n t ~ s y s t e m . ~}$ Note: Image courtesy of Glaukos Corporation.

Postoperatively, patients followed each surgeon's standard postoperative medication regimen, typically consisting of an antibiotic (eg, chloramphenicol) and anti-inflammatory (eg, prednisolone acetate and phenylephrine hydrochloride, AKA prednefrin forte) four times per day for 2 weeks, followed by steroid only twice daily for 2 weeks. Glaucoma medication was discontinued at the treating surgeons' discretion based on the IOP following surgery relative to target IOP.

All data were collected in accordance with the tenets of the Declaration of Helsinki, and all patients signed an informed consent to allow the retrospective evaluation of their clinical data. Descriptive statistics were used to summarize the pre- and postoperative data. Preoperative IOP vs Month 12 IOP were compared using a paired $t$-test, while the McNemar test was used to compare pre- and postoperative proportions of eyes on 0 medications or eyes on $\geq 2$ medications. Results were considered significant for $P$-values $<0.05$. Patients have been followed for 12 months, and follow-up is ongoing.

\section{Results \\ IOP and medications}

A total of 290 eyes were subjected to cataract surgery combined with iStent inject trabecular micro-bypass implantation. At the time of data collection, 165 eyes had postoperative follow-up data through 12 months and constitute the cohort presented in this report. Baseline demographic and ocular characteristics are shown in Table 1. All but three eyes were from Caucasian patients, with the predominant diagnosis being POAG (69.7\%). The most prevalent other diagnoses included OHT (10.9\%), appositional ACG or ACG suspects (6.7\%), PXG (4.8\%), and NTG (4.2\%). One-third of eyes $(55 / 165)$ had undergone prior glaucoma surgery, and $\sim 88 \%$ had mild or moderate glaucoma based on VF criteria (per Hodapp-Anderson-Parrish grading scale).

As detailed in Figure 2 and Table 2, mean IOP reduced significantly from $18.27 \pm 5.41 \mathrm{mmHg}$ preoperatively to $14.04 \pm 2.98 \mathrm{mmHg}$ at 12 months $(23.2 \%$ reduction, $P<0.001)$. All but seven eyes (95.8\% or 158/165) achieved Month 12 IOP of $\leq 18 \mathrm{mmHg}$. The seven remaining eyes, while not meeting the $\leq 18 \mathrm{mmHg}$ cutoff, were able to reduce medications considerably (elimination of 1-2 medications vs preoperative).

Figures 3 and 4 and Table 3 summarize medication burden after stent-cataract surgery. Postoperative mean number of glaucoma medications decreased to $0.47 \pm 0.95$ vs $1.65 \pm 1.28$ preoperatively ( $71.5 \%$ reduction); $76.4 \%$ of eyes $(126 / 165)$ were on zero medications (vs $29 / 165$ or $17.6 \%$ preoperatively; $P<0.001) ; 14.5 \%$ of eyes (24/165) were on $\geq 2$ medications (vs $77 / 165$ or $46.7 \%$ preoperatively; $P<0.001)$; and all but three eyes $(98.2 \%$ or $162 / 165)$ maintained or had reduced medication burden vs their preoperative regimen. The three remaining eyes had low medication burden at baseline (0-1 medication), and substantial IOP reductions were achieved in all, postoperatively (IOP reduced by $4-10 \mathrm{mmHg}$ vs preoperative).

\section{Safety}

Two stents were successfully implanted in all but two eyes $(163 / 165)$ in this study. In the two eyes receiving one stent, IOP reduction at 12 months vs preoperative was still achieved (from $18 \mathrm{mmHg}$ to $14 \mathrm{mmHg}$ in one eye, and from $26 \mathrm{mmHg}$ to $21 \mathrm{mmHg}$ in the other eye). There were no intraoperative complications related to stent implantation. Postoperative adverse events included corneal edema (two cases) and anterior chamber inflammation (two cases) in the immediate postoperative period that resolved by 1 week postoperatively; one case of intermittent stent-iris touch that resulted in no sequelae; one case of postoperative hyphema that resolved by the next visit; and one eye with incomplete visualization of the second stent that resulted in no complications. There were no occurrences of stent obstruction, peripheral anterior synechiae (PAS), or IOP spike $\geq 30 \mathrm{mmHg}$ in any eye during follow-up. Nearly all eyes (97.3\%) achieved CDVA of 20/40 or better at 1 year, stable from $92.7 \%$ preoperatively. There was no appreciable change in average CDR from baseline $(0.69 \pm 0.17)$ to 12 months $(0.68 \pm 0.18)$, nor in VF mean deviation (MD) from baseline $(-5.31 \pm 6.62)$ to 12 months $(-5.50 \pm 7.20)$. Proportional analysis revealed that among eyes with VF data at both the preoperative and 12-month 
Table I Demographic and preoperative characteristics

\begin{tabular}{|c|c|}
\hline Characteristics & $N=165$ \\
\hline \multicolumn{2}{|l|}{ Age (years) } \\
\hline Mean \pm SD & $71.4 \pm 7.6$ \\
\hline Range & $41-88$ \\
\hline \multicolumn{2}{|l|}{ Gender } \\
\hline Male/female & $70 / 95$ \\
\hline \multicolumn{2}{|l|}{ Eye } \\
\hline OD/OS & $75 / 90$ \\
\hline \multicolumn{2}{|l|}{ Cup-to-disc ratio } \\
\hline Mean (SD) & $0.7 \pm 0.2$ \\
\hline Range & $0.1-1.0$ \\
\hline \multicolumn{2}{|l|}{ Prior glaucoma surgical intervention(s) } \\
\hline$\%(n / N)$ & $33.3(55 / 165)$ \\
\hline \multicolumn{2}{|l|}{ CDVA $20 / 40$ or better } \\
\hline$\%(n / N)$ & $92.7(153 / 165)$ \\
\hline \multicolumn{2}{|l|}{ VF mean deviation $(\mathrm{dB})$} \\
\hline Mean (SD) & $-5.3 \pm 6.6$ \\
\hline Range & $-29.0-4.1$ \\
\hline \multicolumn{2}{|l|}{ VF pattern SD (dB) } \\
\hline Mean (SD) & $5.3 \pm 4.0$ \\
\hline Range & $1.0-15.7$ \\
\hline \multicolumn{2}{|l|}{ Central corneal thickness $(\mu \mathrm{m})$} \\
\hline Mean (SD) & $536.1 \pm 37.6$ \\
\hline Range & $405-658$ \\
\hline \multicolumn{2}{|l|}{ Race } \\
\hline$\%(n / N)$ & \\
\hline White & $98.2(162 / 165)$ \\
\hline Black or African-Australian & $0.0(0 / 165)$ \\
\hline Hispanic & $1.2(2 / 165)$ \\
\hline Asian & $0.6(I / 165)$ \\
\hline Other & $0.0(0 / 165)$ \\
\hline \multicolumn{2}{|l|}{ Type of glaucoma } \\
\hline$\%(n / N)$ & \\
\hline Primary open-angle glaucoma & $69.7(115 / 165)$ \\
\hline Ocular hypertension & $10.9(18 / 165)$ \\
\hline Appositional angle-closure glaucoma (ACG) or & $6.7(1 \mathrm{I} / 165)$ \\
\hline ACG suspects & $4.8(8 / 165)$ \\
\hline Pseudoexfoliative glaucoma & $4.2(7 / 165)$ \\
\hline Normal-tension glaucoma & $1.8(3 / 165)$ \\
\hline Combined-mechanism glaucoma & $1.8(3 / 165)$ \\
\hline Pigmentary glaucoma & \\
\hline \multicolumn{2}{|l|}{ Glaucoma severity } \\
\hline$\%(n / N)$ & \\
\hline Mild & $69.9(109 / 156)$ \\
\hline Moderate & $17.9(28 / 156)$ \\
\hline Severe & $12.2(19 / 156)$ \\
\hline
\end{tabular}

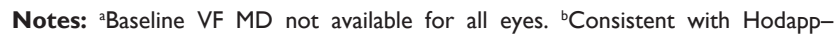
Anderson-Parrish visual field criteria. Mild: VF MD no worse than $-6 \mathrm{~dB}$, moderate: VF MD worse than $-6 \mathrm{~dB}$ but no worse than $-12 \mathrm{~dB}$, severe: VF MD worse than $-12 \mathrm{~dB}$.

Abbreviations: CDVA, corrected distance visual acuity; MD, mean deviation; $\mathrm{VF}$, visual field. visits ( $\mathrm{n}=131), 89.3 \%(117 / 131)$ had either improvement, no change, or difference of less than $3 \mathrm{~dB}$ in their VF MD (less than half the SD for MD measurement, ie, within the margin of error of the test).

Additional glaucoma procedures were undertaken in three eyes during the follow-up period. These procedures included one eye with deep sclerectomy plus mitomycin C (MMC), one eye with a second iStent inject implantation, and one eye with selective laser trabeculoplasty at 3 months and trabeculectomy plus MMC at 6 months postoperatively. All three eyes had history of glaucoma surgery prior to iStent inject-cataract surgery.

\section{Discussion}

The current study results support the effectiveness and safety of the iStent inject system in conjunction with cataract surgery for the treatment of various types of mild to advanced glaucoma or OHT. Both mean IOP and mean medication use decreased significantly from baseline to the 12-month visit, while post-phacoemulsification CDVA improvement was maintained and safety parameters such as CDR and VF remained stable. To our knowledge, this is the first large multicenter, multi-provider study of the performance of this microstent in an Asia-Pacific population within a routine clinical setting.

Unlike randomized controlled trials where the characteristics of the patient population are well-defined by inclusion/exclusion criteria, the current study population was heterogeneous in their diagnoses and disease burden. As such, the outcomes in this cohort would be expected to be more generalizable to current practicing ophthalmologists with diverse patient populations. In this study, patients ranged from those with no previous glaucoma surgery or medical treatments, to those with prior glaucoma surgery and uncontrolled IOP and/or three or more medications. Given this range of disease burden, it is appropriate to assess outcomes in the context of a patient's entire clinical picture, taking into account both IOP and medications. For example, in eyes with relatively well-controlled medicated IOP preoperatively, a key expectation for surgery may be to reduce medications, while also maintaining or lowering IOP. In eyes with uncontrolled IOP despite numerous medications, an expectation for surgery may be to reduce IOP, while also maintaining or reducing medications. In our cohort, over $98 \%$ of eyes maintained or decreased their medication burden at 12 months vs preoperative; the remaining eyes, although not reducing medications, achieved substantial IOP 


\section{$23.2 \%$ IOP reduction at M12 vs preoperative $(P<0.001)$}

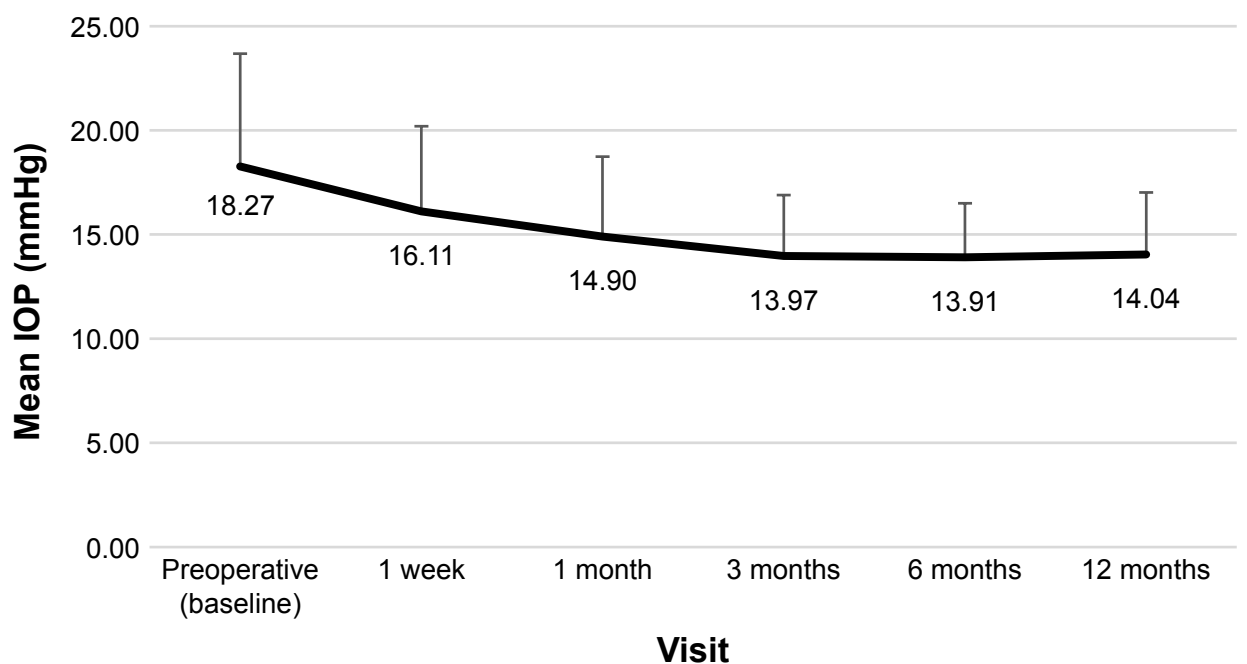

Figure 2 Reduction in mean intraocular pressure (IOP) through 12 months postoperatively. Note: Available eyes at each visit $(\mathrm{N}=165)$

reduction postoperatively. Likewise, over $95 \%$ of eyes had IOP $\leq 18 \mathrm{mmHg}$ at 12 months; the remaining seven eyes, although not reaching the $\leq 18 \mathrm{mmHg}$ IOP cutoff, achieved considerable medication reduction postoperatively. Regardless of preoperative IOP and medication burden, all patients needed cataract surgery and thus iStent inject offered an opportunity to address IOP with minimal to no additional patient burden beyond cataract surgery itself.

The outcomes of this study are directionally and quantitatively consistent with those of prior studies of iStent inject in conjunction with cataract surgery. For example, ArriolaVillalobos et a $\mathrm{l}^{18}$ followed a group of patients after combined cataract surgery and iStent inject implantation. At 1 year, IOP reduced from $19.95 \pm 3.71 \mathrm{mmHg}$ to $16.75 \pm 2.24 \mathrm{mmHg}$, and mean number of glaucoma medications decreased from $1.3 \pm 0.66$ to $0.3 \pm 0.57$. Overall, $100 \%(20 / 20)$ of patients demonstrated a $\geq 20 \%$ decrease in IOP from baseline, and
$75 \%(15 / 20)$ of eyes became medication-free. The percentage IOP decrease in the present study also is consistent with prior studies of one or more iStents with cataract surgery in which the percent change in mean IOP from baseline to 12 months ranged from $\sim 10 \%$ to $40 \%{ }^{2,3,19-22}$ The $23.2 \%$ reduction in mean IOP was statistically significant and occurred despite a relatively low starting IOP $(18.27 \mathrm{mmHg})$, a lack of a preoperative washout period, and inclusion of eyes with NTG.

Medication non-adherence is a well-known issue for patients on glaucoma drops, affecting the great majority of medicated eyes. ${ }^{7-9,23}$ In particular, the increase from single to multiple drops has been shown to dramatically decrease adherence, as demonstrated in the study by Robin and Covert ${ }^{24}$ using prescription refills as a proxy for adherence. In the current study, the percentage of eyes on two or more medications decreased by more than two-thirds $(46.7 \%$ of

Table 2 Proportional analysis of postoperative IOP of available eyes at each visit $(\mathrm{N}=165)$ through 12 months

\begin{tabular}{|c|c|c|c|c|c|c|}
\hline \multirow[t]{2}{*}{ IOP outcome } & Preop & WI & MI & M3 & M6 & MI2 \\
\hline & n (\%) & n (\%) & n (\%) & n (\%) & n (\%) & n (\%) \\
\hline Available at visit & 165 & 155 & 163 & 148 & 150 & 165 \\
\hline \multirow[t]{2}{*}{$\mathrm{IOP} \leq 15 \mathrm{mmHg}$} & 59 & 71 & 104 & 114 & 112 & 111 \\
\hline & $(35.76)$ & $(45.81)$ & $(63.80)$ & $(77.03)$ & $(74.67)$ & $(67.27)$ \\
\hline \multirow[t]{2}{*}{$\mathrm{IOP} \leq 18 \mathrm{mmHg}$} & 100 & 116 & 140 & 142 & 145 & 158 \\
\hline & $(60.6 \mathrm{I})$ & $(74.84)$ & $(85.89)$ & $(95.95)$ & $(96.67)$ & $(95.76)$ \\
\hline \multirow[t]{2}{*}{ IOP decreased $\geq 20 \%$ vs preop IOP } & & 52 & 79 & 81 & 82 & 85 \\
\hline & & $(33.55)$ & $(48.47)$ & $(54.73)$ & $(54.67)$ & $(51.52)$ \\
\hline
\end{tabular}

Abbreviations: $M$, month; Preop, preoperative; W, week; IOP, intraocular pressure. 


\section{$71.5 \%$ reduction in number of medications at month 12 vs preoperative}

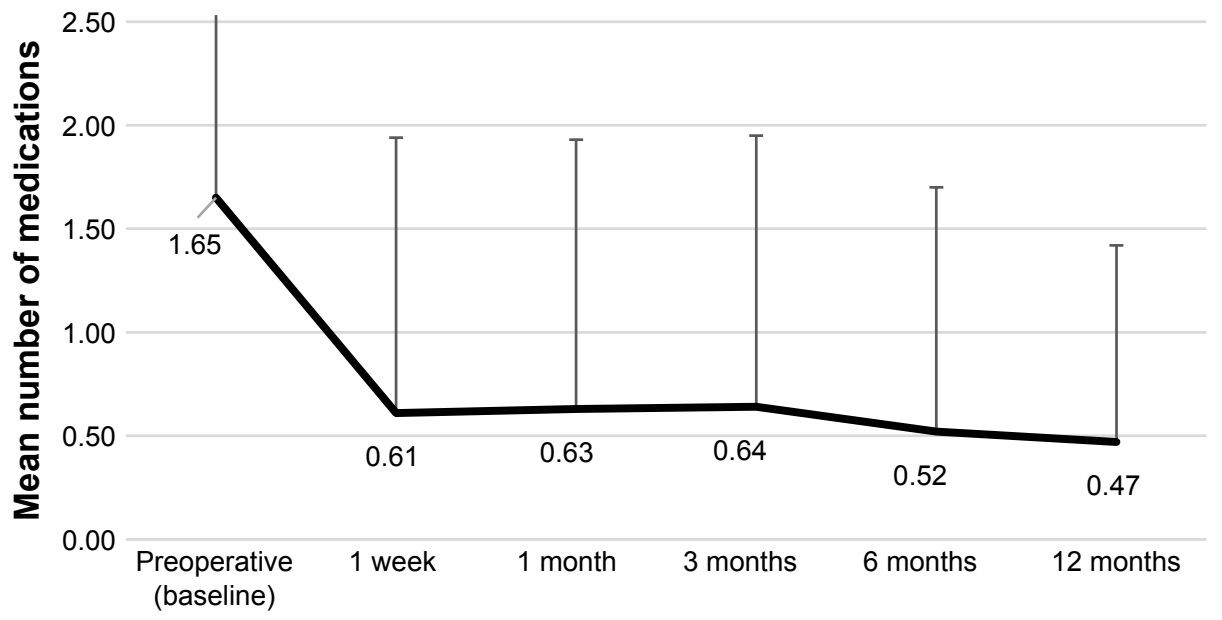

Visit

Figure 3 Reduction in mean number of glaucoma medications through 12 months postoperatively. Note: Available eyes at each visit $(\mathrm{N}=165)$.

eyes preoperatively vs $14.5 \%$ at 12 months; $P<0.001)$. Conversely, the proportion of eyes on no medications more than quadrupled (from $17.6 \%$ preoperatively to $76.4 \%$ at 12 months; $P<0.001)$. Nearly all eyes had the same or decreased medication burden vs their preoperative regimen. These medication reductions underscore the patient-level benefit of iStent inject combined with cataract surgery as a glaucoma treatment that reliably reduces the number of existing medications and itself does not rely on patient adherence.
The percent decrease in medication burden vs baseline $(\sim 60 \%-100 \%)$ in prior studies of iStent and iStent inject is compelling, ${ }^{2-4,12-16,18-22,25-27}$ and is consistent with the $71.5 \%$ medication decrease observed in the current study. This medication reduction is particularly noteworthy given that this study included all patients undergoing implantation at the sites involved (including patients with NTG or OHT who are not taking medication), as well as a lower mean baseline medication burden (mean 1.65 preoperative medications in

$76.4 \%$ of eyes medication-free vs $17.6 \%$ preoperatively $(P<0.001)$

$14.5 \%$ of eyes on $\geq 2$ medications vs $46.7 \%$ preoperatively $(P<0.001)$

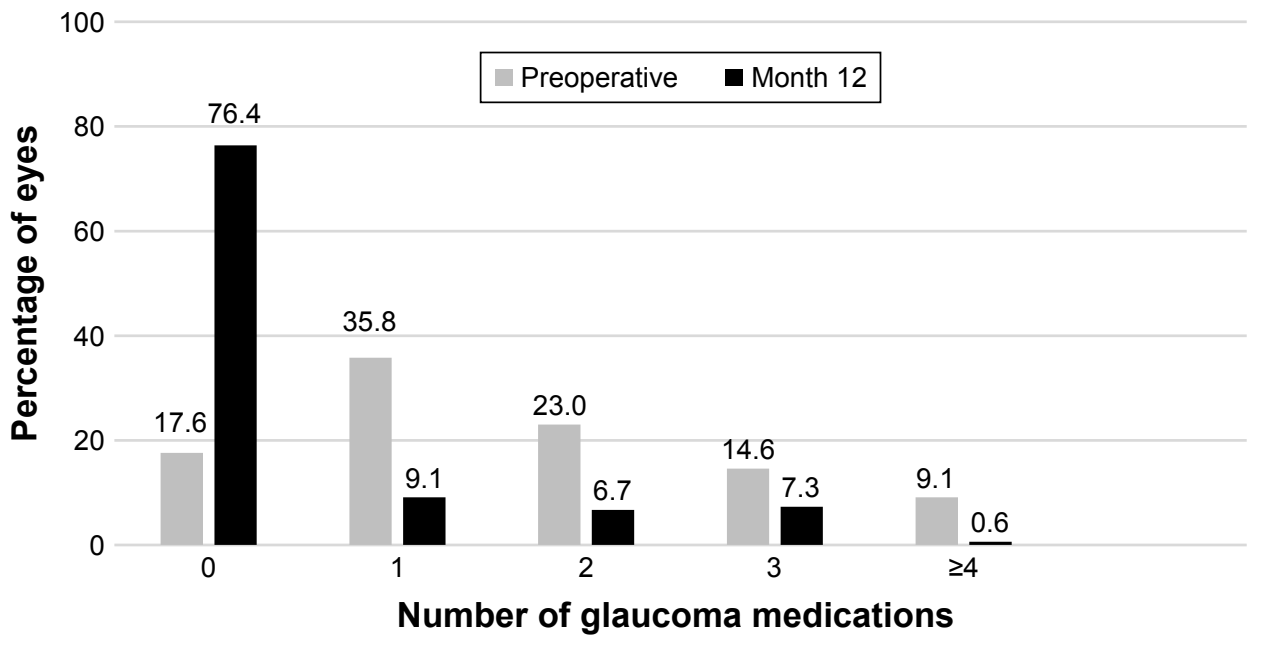

Figure 4 Reduction in glaucoma medication burden at 12 months postoperatively. Note: Available eyes at each visit $(\mathrm{N}=165)$. 
Table 3 Proportional analysis of medication burden of available eyes at each visit through 12 months $(\mathrm{N}=165)$

\begin{tabular}{|c|c|c|c|c|c|c|}
\hline \multirow[t]{2}{*}{ Medication change vs preop } & Preop & WI & MI & M3 & M6 & MI2 \\
\hline & n (\%) & n (\%) & n (\%) & n (\%) & n (\%) & n (\%) \\
\hline Available at visit & 165 & 155 & 163 & 146 & $15 \mid$ & 165 \\
\hline \multirow[t]{2}{*}{ No change in med from preop } & & 51 & 56 & 43 & 43 & 39 \\
\hline & & $(32.90)$ & $(34.36)$ & $(29.45)$ & $(28.48)$ & $(23.64)$ \\
\hline \multirow[t]{2}{*}{ Increase in med from preop } & & I & 1 & 1 & 2 & 3 \\
\hline & & $(0.65)$ & $(0.61)$ & $(0.68)$ & $(1.32)$ & $(1.82)$ \\
\hline \multirow[t]{2}{*}{ Decrease in med from preop } & & 103 & 106 & 102 & 1.6 & 123 \\
\hline & & $(66.45)$ & $(65.03)$ & $(69.86)$ & $(70.20)$ & $(74.55)$ \\
\hline
\end{tabular}

Abbreviations: $M$, month; preop, preoperative; W, week; med, medication.

this study). In addition, some eyes had advanced glaucoma (VF MD worse than $-12 \mathrm{~dB}$ ) with low IOP targets; so it is likely surgeons elected to continue medical treatment in an attempt to achieve maximum IOP reduction in these cases.

In addition, it is widely acknowledged that cataract surgery itself may decrease IOP through the 1-year visit. ${ }^{28}$ The decrease is small, $\sim 1-2 \mathrm{mmHg}$ for any given eye, ${ }^{28}$ and is expected to be lower with lower baseline IOP $(\leq 20 \mathrm{mmHg}){ }^{29,30}$ Since the mean baseline IOP in the current study was relatively low $(18.27 \pm 5.41 \mathrm{mmHg})$, and since the patients comprised a wide range of preoperative disease severity, a decrease in the overall mean may have been difficult to detect. However within this cohort, IOP decreased by more than $4 \mathrm{mmHg}$, more than twice the amount expected from cataract surgery alone. This is consistent with clinical studies showing significantly greater reductions in IOP and medication use in patients with iStent implantation in conjunction with cataract surgery compared to patients undergoing cataract surgery alone..$^{2-4,31}$ It also aligns with the work of Fernandez-Barrientos et $\mathrm{al}^{2}$ who demonstrated significant increases in trabecular outflow facility at 12 months in eyes implanted with iStent with cataract surgery compared to eyes with cataract surgery alone. More recently, iStent inject has been the subject of a large prospective, randomized, controlled, multicenter pivotal clinical trial. In that study, iStent inject-phacoemulsification eyes demonstrated statistically and clinically significant benefit over phacoemulsification alone: specifically, higher proportion of eyes achieving $\geq 20 \%$ reduction at 24 months from baseline in unmedicated diurnal IOP; higher proportion of eyes with Month 24 unmedicated IOP $\leq 18 \mathrm{mmHg}$; and higher proportion of eyes achieving freedom from medications. ${ }^{32}$

The safety profile of iStent inject surgery was excellent. No stent-related intraoperative complications occurred. Postoperative adverse events were transient, few in number, and not associated with sequelae. Notably, there were no cases of stent obstruction or PAS, in contrast to the rates seen in other recent MIGS studies. ${ }^{33,34}$ Nearly all eyes (97.3\%) achieved CDVA of 20/40 or better at 1 year. Mean CDR and VF also remained stable, with the vast majority of eyes experiencing minimal to no decline in VF MD from baseline to 12 months.

Several limitations exist in this retrospective study. As is the case in most real-life clinical cohorts, it was not appropriate to complete medication washout due to the potential risks of IOP spikes in participating eyes. Medications were managed according to each surgeon's routine clinical practice; although these management choices generally were consistent across the five surgeons, they did not follow a formal protocol as might be the case in a randomized clinical trial. No diurnal IOP measurements were obtained, leaving open the possibility of regression to the mean. Since CDR can be impacted by optic nerve size, variable grading could arise; however, any change over time still would be apparent since the measurements were completed in the same patients and by the same surgeons over the course of the study. At the time of data collection, $80 \%$ of eyes had completed VF testing; future reports from this cohort could aim to include more comprehensive VF data. In regards to the treatment plan, the cost-benefit balance between lower IOP and higher medication burden may be decided differently by different investigators or patients, which could bias study results. There was no formal control group of cataract surgery alone, and thus it was not possible to conclusively isolate the effects of the stents vs those of cataract surgery; however, other prospective comparative trials of iStent-phaco or iStent inject-phaco vs phaco alone - $^{2-431,32}$ can be used as a reference point. iStent inject is a relatively new technology in Australia, so future reports may assess longer-term outcomes and larger sample sizes. Other investigations could include subgroup analyses of eyes with different glaucoma types or stratification by history of prior glaucoma surgery. 


\section{Conclusion}

This report presents the largest multicenter, multi-surgeon cohort to date within an Asia-Pacific clinical setting. Additionally, it includes data on eyes that often are excluded from MIGS studies, such as those with PXG, appositional ACG, normotensive glaucoma, advanced glaucoma, and OHT, and it documents these outcomes in real-life, clinically relevant treatment scenarios. Outcomes show that the iStent inject can produce significant and safe IOP and medication reductions in various types of mild to advanced glaucoma, and that cataract surgery may provide a propitious opportunity to employ this technology in managing glaucoma.

\section{Acknowledgments}

This work was presented in part at the 2018 annual meeting of the Asia Pacific Glaucoma Congress (APGC) and at the 2018 International Congress of Glaucoma Surgery. No financial support was given for the work in this study. Editorial assistance and publication processing charges were provided by Glaukos Corporation.

\section{Disclosure}

CIC reports travel grant, speaker and advisory board fees from Glaukos. DM reports personal fees and non-financial support from Glaukos during the conduct of the study; and personal fees from Alcon and Allergan, and speaker fees from Johnson and Johnson, outside the submitted work. The authors report no other conflicts of interest in this work.

\section{References}

1. Pillunat LE, Erb C, Jünemann AG, Kimmich F. Micro-invasive glaucoma surgery (MIGS): a review of surgical procedures using stents. Clin Ophthalmol. 2017;11:1583-1600.

2. Fernández-Barrientos Y, García-Feijoó J, Martínez-de-La-Casa JM, Pablo LE, Fernández-Pérez C, García Sánchez J. Fluorophotometric study of the effect of the glaukos trabecular microbypass stent on aqueous humor dynamics. Invest Ophthalmol Vis Sci. 2010;51(7):3327-3332.

3. Samuelson TW, Katz LJ, Wells JM, Duh YJ, Giamporcaro JE; US iStent Study Group. Randomized evaluation of the trabecular micro-bypass stent with phacoemulsification in patients with glaucoma and cataract. Ophthalmology. 2011;118(3):459-467.

4. Fea AM. Phacoemulsification versus phacoemulsification with microbypass stent implantation in primary open-angle glaucoma: randomized double-masked clinical trial. J Cataract Refract Surg. 2010;36(3): 407-412.

5. Leung EW, Medeiros FA, Weinreb RN. Prevalence of ocular surface disease in glaucoma patients. J Glaucoma. 2008;17(5):350-355.

6. Baudouin C, Liang H, Hamard P, et al. The ocular surface of glaucoma patients treated over the long term expresses inflammatory markers related to both T-helper 1 and T-helper 2 pathways. Ophthalmology. 2008; 115(1):109-115.

7. Nordstrom BL, Friedman DS, Mozaffari E, Quigley HA, Walker AM. Persistence and adherence with topical glaucoma therapy. Am J Ophthalmol. 2005;140(4):598-606.
8. Friedman DS, Quigley HA, Gelb L, et al. Using pharmacy claims data to study adherence to glaucoma medications: methodology and findings of the glaucoma adherence and persistency study (GAPs). Invest Ophthalmol Vis Sci. 2007;48(11):5052-5057.

9. Robin AL, Novack GD, Covert DW, Crockett RS, Marcic TS. Adherence in glaucoma: objective measurements of once-daily and adjunctive medication use. Am J Ophthalmol. 2007;144(4):533-540.

10. Ansari E. An update on implants for minimally invasive glaucoma surgery (MIGS). Ophthalmol Ther. 2017;6(2):233-241.

11. Vinod K, Gedde SJ, Feuer WJ, et al. Practice preferences for glaucoma surgery: a survey of the American glaucoma Society. J Glaucoma. 2017;26(8):687-693.

12. Berdahl J, Voskanyan L, Myers JS, et al. Implantation of two secondgeneration trabecular micro-bypass stents and topical travoprost in open-angle glaucoma not controlled on two preoperative medications: 18-month follow-up. Clin Exp Ophthalmol. 2017;45(8):797-802.

13. Fea AM, Belda JI, Rękas M, et al. Prospective unmasked randomized evaluation of the iStent inject (®) versus two ocular hypotensive agents in patients with primary open-angle glaucoma. Clin Ophthalmol. 2014; 8:875-882.

14. Klamann MK, Gonnermann J, Pahlitzsch M, et al. iStent inject in phakic open angle glaucoma. Graefes Arch Clin Exp Ophthalmol. 2015;253(6):941-947.

15. Lindstrom R, Lewis R, Hornbeak DM, et al. Outcomes following implantation of two second-generation trabecular Micro-Bypass stents in patients with open-angle glaucoma on one medication: 18-month follow-up. Adv Ther. 2016;33(11):2082-2090.

16. Voskanyan L, García-Feijoó J, Belda JI, et al. Prospective, unmasked evaluation of the iStent ${ }^{\circledR}$ inject system for open-angle glaucoma: synergy trial. Adv Ther. 2014;31(2):189-201.

17. Pager CK. Expectations and outcomes in cataract surgery: a prospective test of 2 models of satisfaction. Arch Ophthalmol. 2004;122(12): $1788-1792$

18. Arriola-Villalobos P, Martinez-de-La-Casa JM, Diaz-Valle D, Morales-Fernandez L, Fernandez-Perez C, Garcia-Feijoo J. Glaukos iStent inject ${ }^{\mathbb{B}}$ trabecular Micro-Bypass implantation associated with cataract surgery in patients with coexisting cataract and open-angle glaucoma or ocular hypertension: a long-term study. J Ophthalmol. 2016;2016(3):1-7.

19. Fea AM, Consolandi G, Zola M, et al. Micro-Bypass implantation for primary open-angle glaucoma combined with phacoemulsification: 4-year follow-up. J Ophthalmol. 2015;2015:795357.

20. Neuhann TH. Trabecular micro-bypass stent implantation during small-incision cataract surgery for open-angle glaucoma or ocular hypertension: long-term results. J Cataract Refract Surg. 2015;41(12): 2664-2671.

21. Belovay GW, Naqi A, Chan BJ, Rateb M, Ahmed II. Using multiple trabecular micro-bypass stents in cataract patients to treat open-angle glaucoma. J Cataract Refract Surg. 2012;38(11):1911-1917.

22. Arriola-Villalobos P, Martínez-de-la-Casa JM, Díaz-Valle D, Fernández-Pérez C, García- Sánchez J, García-Feijoó J. Combined iStent trabecular micro-bypass stent implantation and phacoemulsification for coexistent open-angle glaucoma and cataract: a long-term study. Br J Ophthalmol. 2012;96(5):645-649.

23. Tsai JC. A comprehensive perspective on patient adherence to topical glaucoma therapy. Ophthalmology. 2009;116(11 Suppl):S30-S36.

24. Robin A, Covert D. Does adjunctive glaucoma therapy affect adherence to the initial primary therapy? Ophthalmology. 2005;112(5):863-868.

25. Vold SD, Voskanyan L, Tetz M, et al. Newly diagnosed primary open-angle glaucoma randomized to 2 trabecular bypass stents or prostaglandin: outcomes through 36 months. Ophthalmol Ther. 2016;5(2): 161-172.

26. Arriola-Villalobos P, Martínez-de-La-Casa JM, Díaz-Valle D, et al. Mid-term evaluation of the new Glaukos iStent with phacoemulsification in coexistent open-angle glaucoma or ocular hypertension and cataract. Br J Ophthalmol. 2013;97(10):1250-1255. 
27. Craven ER, Katz LJ, Wells JM, Giamporcaro JE; iStent Study Group. Cataract surgery with trabecular micro-bypass stent implantation in patients with mild-to-moderate open-angle glaucoma and cataract: two-year follow-up. J Cataract Refract Surg. 2012;38(8):1339-1345.

28. Vizzeri G, Weinreb RN. Cataract surgery and glaucoma. Curr Opin Ophthalmol. 2010;21(1):20-24.

29. Poley BJ, Lindstrom RL, Samuelson TW. Long-term effects of phacoemulsification with intraocular lens implantation in normotensive and ocular hypertensive eyes. J Cataract Refract Surg. 2008;34(5): 735-742.

30. Shingleton BJ, Gamell LS, O’Donoghue MW, Baylus SL, King R. Long-term changes in intraocular pressure after clear corneal phacoemulsification: normal patients versus glaucoma suspect and glaucoma patients. J Cataract Refract Surg. 1999;25(7):885-890.

31. El Wardani M, Bergin C, Achache F, Sharkawi E. Evaluating the trabecular micro-bypass stent combined with phacoemulsification compared to phacoemulsification alone. Klin Monbl Augenheilkd. 2015; 232(4):442-445
32. Samuelson TW. Prospective, Randomized, Multicenter Clinical Investigation of the Glaukos iStent inject. Platform presentation at: Annual meeting of the American Society of Cataract and Refractive Surgeons; April 13-17; 2018; Washington, DC.

33. Pfeiffer N, Garcia-Feijoo J, Martinez-de-La-Casa JM, et al. A randomized trial of a Schlemm's canal Microstent with phacoemulsification for reducing intraocular pressure in open-angle glaucoma. Ophthalmology. 2015;122(7):1283-1293.

34. Samuelson TW, Chang DF, Marquis R, et al. A Schlemm canal Microstent for intraocular pressure reduction in primary open-angle glaucoma and cataract: the horizon study. Ophthalmology. 2019;126(1): 29-37.
Clinical Ophthalmology

\section{Publish your work in this journal}

Clinical Ophthalmology is an international, peer-reviewed journal covering all subspecialties within ophthalmology. Key topics include: Optometry; Visual science; Pharmacology and drug therapy in eye diseases; Basic Sciences; Primary and Secondary eye care; Patien Safety and Quality of Care Improvements. This journal is indexed on

Submit your manuscript here: http://www.dovepress.com/clinical-ophthalmology-journal

\section{Dovepress}

PubMed Central and CAS, and is the official journal of The Society of Clinical Ophthalmology (SCO). The manuscript management system is completely online and includes a very quick and fair peer-review system, which is all easy to use. Visit http://www.dovepress.com/ testimonials.php to read real quotes from published authors. 